\title{
Knowledge about Osteoporosis Risk Prevention in Young and Post-Menopausal Women in Palmerston North, New Zealand ${ }^{+}$
}

\author{
E. Reynolds ${ }^{1}$, K. Schraders ${ }^{1}$, G. Zatta ${ }^{1}$, J. Weber ${ }^{1}$, J. Coad ${ }^{1}$, M. Kruger ${ }^{1,2,3}$ and J. Thomson ${ }^{1, *}$ \\ 1 School of Food and Advanced Technology, College of Sciences, Massey University, Palmerston North, \\ 4442, New Zealand; E.Reynolds@massey.ac.nz (E.R.); K.Schraders@massey.ac.nz (K.S.); \\ Giancarlazatta@gmail.com (G.Z.); J.L.Weber@massey.ac.nz (J.W.); J.Coad@massey.ac.nz (J.C.); \\ M.C.Kruger@massey.ac.nz (M.K.) \\ 2 College of Health, Massey University, Palmerston North, 4442, New Zealand \\ 3 Riddet Institute, Massey University, Palmerston North, 4442, New Zealand \\ * Correspondence: J.A.Thomson@massey.ac.nz \\ + Presented at the 2018 Nutrition Society of New Zealand Annual Conference, Auckland, New Zealand, \\ 28-30 November 2018.
}

Published: 12 March 2019

Background: Critical determinants of bone health are genetics, diet and physical activity. Women face challenges to the modifiable risk factors. Young women, who have not completed bone mineral deposition, often don't meet the dietary recommendations. Post-menopausal women, who have marked bone loss, often don't meet the calcium recommendations, which are higher for this age group, and physical activity recommendations. Others have found knowledge of osteoporosis differs between young and older women. Therefore, this study investigated women's knowledge about osteoporosis at two life stages important for bone health.

Methods: Ninety-nine healthy young women (18-26 y) and 50 post-menopausal women completed the Revised Osteoporosis Knowledge Test (OKT). Minor changes were made to the OKT, researchers removed one question on overweight so as not to influence participants' answers to another questionnaire, and two on Recommended Dietary Intake for calcium and vitamin D, as these questions were difficult and not relevant to layperson's knowledge about osteoporosis.

Results: Item score distributions were non-parametric, except total scores were normally distributed, determined by Kolmogorov-Smirnov test. The women had moderate knowledge about osteoporosis (correct scores were median: 58.6\%). Median knowledge did not differ in risk, exercise, or nutrition items $(p>0.05)$; however, the older women had significantly greater mean rank scores in the osteoporosis diagnosis and treatment item (median, $(\mathrm{Q} 1, \mathrm{Q} 3)$ young; $2(1,2)$, post-menopausal women; $2(2,3), p=0.002)$, determined by Mann-Whitney U. Interestingly, all women had moderate to high knowledge about calcium-rich food sources (median 80.8\%), and the older women had significantly greater mean rank scores (young; $4(3,4)$, post-menopausal; $4(3,5), p=0.010$. Further investigation of the health beliefs and self-efficacy of these populations is warranted to better understand the challenges to meeting calcium and physical activity recommendations.

Conclusions: These women demonstrated moderate knowledge of osteoporosis risk, however older women were more aware of the diagnosis and treatment of osteoporosis than younger women.

(C) 2019 by the authors. Licensee MDPI, Basel, Switzerland. This article is an open access article distributed under the terms and conditions of the Creative Commons Attribution (CC BY) license (http://creativecommons.org/licenses/by/4.0/). 